\title{
Radiological Underestimation of Tumor Size Influences the Success Rate of Re-Excision after Breast-conserving Surgery
}

(1) Duncan Simpson, (1) Jennifer Allan, (1) Brendan McFall

Breast Unit, Antrim Area Hospital, Bush Road, Antrim, Northern Ireland, United Kingdom

\begin{abstract}
Objective: Failure to achieve adequate margins after breast-conserving surgery often leads to re-excision, either by repeat breast-conserving surgery (BCS) or by mastectomy. Despite the high frequency of this problem, the success rate of achieving adequate margins by repeat BCS is not well documented. The objective of this study was to determine the success rate of repeat BCS and identify the factors influencing that rate.

Materials and Methods: A retrospective review was performed of all women undergoing repeat BCS for inadequate margins after initial BCS in our breast unit between 2013 and 2019. Univariate and multivariate analyses were carried out to identify the factors influencing how often adequate margins were achieved after repeat BCS.

Results: One hundred fifty-four patients underwent repeat BCS after initially inadequate margins, of which adequate margins were achieved in $82 \%$. Patients with successful repeat BCS had smaller tumors, had less underestimation of tumor size on imaging, and were less likely to have had cavity shaves taken at their initial BCS. A tumor size more than 50\% larger than predicted by imaging was independently associated with failure of repeat BCS in multivariate analysis (odds ratio: $3.6,95 \%$ CI: $1.41-9.20, \mathrm{p}=0.007$ ). Underestimation of tumor size by imaging was commoner and more extensive in patients with larger tumors and those with ductal carcinoma in situ.
\end{abstract}

Conclusion: Re-excision by cavity shaves has a high success rate and should be offered to all patients who are deemed suitable for the procedure. Patients whose tumors are more than 50\% larger than predicted by imaging should be counseled about the higher risk of failure.

Keywords: Breast-conserving surgery, breast imaging, breast neoplasms, margins of excision, re-excision

Cite this article as: Simpson D, Allan J, McFall B. Radiological Underestimation of Tumour Size Influences Success Rate of Re-Excision After Breast Conserving Surgery. Eur J Breast Health 2021; 17(4): 363-370.

\section{Key Points}

- In patients in whom initial breast-conserving surgery has not achieved adequate margins, repeat breast-conserving surgery is successful in $82 \%$ of cases.

- Underestimation of tumor size by imaging reduces the probability that repeat breast-conserving surgery will be successful.

- Underestimation of tumor size by imaging is commoner in patients with larger tumors and ductal carcinoma in situ.

\section{Introduction}

Breast-conserving surgery (BCS) is now firmly established as the standard of care for early breast cancer where feasible, with long-term follow-up demonstrating oncological safety (1). With ever-improving adjuvant treatment and understanding of tumor biology, local recurrence rates after BCS are low. Re-excision rates, on the other hand, despite improvement in recent years, remain high (2). With BCS performed on 180,000 women in the USA each year and a significant number of those requiring further surgery to obtain adequate margins, re-excision is clearly an area where improvements could have a significant impact on healthcare delivery (3).

For women requiring re-excision, a decision needs to be made on whether to perform repeat BCS, with further shaves of tissue removed at the inadequate margins, or proceed to mastectomy. Mastectomy guarantees that surgical treatment is complete and delivers an extremely high rate of margin clearance but is associated with an increased risk of short- and long-term morbidity and poorer body image $(4,5)$. Repeat BCS, on the other hand, allows the opportunity for conservation of the breast, although persistent inadequate margins may lead to a third or even a fourth operation, with increased operative risk, increased cost, poorer cosmesis, and a possible delay in adjuvant treatment (6-8). The decision on the type of re-excision is based primarily on the chance of repeat BCS successfully achieving an adequate margin, although the expected cosmetic 
result, the woman's attitude toward the risk of additional surgery, her suitability for an oncoplastic technique, and her degree of aversion to undergoing mastectomy also play a role. Given how often re-excision is necessary, the evidence base addressing factors that affect the success rate of repeat BCS is small.

The aim of this study was to determine the success rate of repeat BCS in achieving adequate margins and to identify clinical factors available at the time when the decision on the method of re-excision is made that would allow us to more accurately define that rate for each individual patient, allowing better informed decision making by patients and their surgeons on the method of re-excision.

\section{Materials and Methods}

All patients undergoing initial BCS in our breast unit between January 2013 and October 2019 were identified from a prospectively compiled database. Those undergoing re-excision by repeat BCS were included in the study. The exclusion criteria were patients undergoing neoadjuvant chemotherapy; patients where the initial BCS was an excision biopsy for diagnosis; patients undergoing repeat BCS by an oncoplastic reduction technique; patients with phyllodes tumors; patients undergoing repeat surgery to the axilla only; and patients undergoing repeat surgery to the breast for multifocal disease, early recurrence, or surgical complications. Approval was given by the local research governance committee. Patients routinely underwent preoperative digital mammography using Hologic Selenia Dimensions (Hologic, Marlborough, MA, USA) and ultrasound using Toshiba Xario (Toshiba, Tokyo, Japan) equipment. Magnetic resonance imaging (MRI) was not used routinely but was employed in scenarios where it was felt likely to alter management, particularly in patients with lobular tumors or with a marked discrepancy in tumor size between the mammography and ultrasonography results. When used, MRI was performed with either GE Optima (GE Healthcare, Chicago, IL, USA) or Siemens Sola (Siemens, Munich, Germany), both 1.5 tesla wide bore with 16 and 18 channel coils, respectively.

The choice of initial surgical approach, either mastectomy or BCS, was made by the multidisciplinary team in conjunction with the patient, taking into account factors such as radiological prediction of tumor size, breast size, tumor biology, genetic status, and comorbidities. The decision on whether to perform re-excision in patients with inadequate margins, as well as whether to achieve this by mastectomy or repeat BCS, was also made by the multidisciplinary team in conjunction with the patient, taking into account factors such as the number of involved margins, pathological tumor size, and perceived cosmetic outcome.

The multidisciplinary team followed the United Kingdom guidelines for adequate margin distance in invasive and non-invasive disease. The minimum adequate margin decreased to $1 \mathrm{~mm}$ during the study period, with the policy of the multidisciplinary team also changing to mirror these guidelines. Patients with an unsatisfactory deep or superficial margin were not routinely re-excised if the initial excision was known to extend to the pectoral fascia or subcutaneous tissue. The technique of planned circumferential cavity shaving in addition to wide local excision was not used in this study. Unplanned targeted cavity shaves were taken during the initial BCS at the operating surgeon's discretion if it was felt that a particular margin was at risk of being involved, either because of visualization or palpation of the tumor at the edge of the wide local excision specimen or breast cavity, or because of concern performed for all wire-localized excisions but not for excisions where the tumor was palpable. No intraoperative pathological assessment of margins was performed.

The potential factors predicting the success of BCS investigated were age at the time of initial surgery; radiological tumor size; presence of ductal carcinoma in situ (DCIS); presence of DCIS only; pathological tumor size; Bloom-Richardson-Elston grade; tumor type; multifocality; axillary lymph node involvement; number of involved radial margins; whether targeted cavity shaves were taken at initial surgery; presence of lymphovascular invasion; estrogen receptor (ER) status; and human epidermal growth factor receptor 2 (HER2) status.

Assessment of the maximum diameter of the radiological malignancy was made by a consultant breast radiologist while performing the breast ultrasound or reporting the images of the mammogram and, if performed, the MRI. The radiological tumor size was defined as the largest of these measurements, irrespective of modality. This measurement was chosen as it is likely to be the measurement used both to decide whether to perform BCS initially and to plan the size of the resection specimen removed if BCS is performed.

Pathological tumor size was defined as the greatest diameter of the whole tumor, including any DCIS, as measured by a consultant pathologist. If tumors were multifocal, the size of the largest focus was used. If any additional tumor was found in targeted cavity shaves at the initial BCS, this was added to the pathological tumor size. Additional tumor removed at the repeat BCS was not added to the pathological tumor size for the purpose of the results of this study as this information is not available at the time when the decision on the method of re-excision is made and so could not contribute to the aims of this study. It was, however, used in determining the total tumor size used in planning the patients' adjuvant treatment.

The pathological tumor size to radiological tumor size ratio (PRR) was used as a measure of the degree of radiological underestimation or overestimation of tumor size. This was calculated by dividing the pathological tumor size by the radiological tumor size. A higher PRR signifies a greater degree of radiological tumor size underestimation.

Patients with Paget's disease of the breast were excluded from analysis involving radiological tumor size. Patients with pure DCIS were excluded from analysis involving grade, ER status, HER2 status, and lymphovascular invasion as these are not routinely recorded in our institution for these patients. Pathology reports from the re-excision specimen were examined for the presence of any DCIS or invasive carcinoma and whether the re-excision had achieved adequate margins.

The authors state that the study protocol has been approved by the Northern Health and Social Care Trust research committee (decision number: NT20-274636-02 date: June 10 ${ }^{\text {th }}, 2020$ ).

\section{Statistical analysis}

Statistical analysis was performed using SPSS (SPSS Statistics for Macintosh, Version 24.0; IBM Corp, Armonk, NY, USA). Continuous variables were assessed by the Student's t-test for parametric data and Mann-Whitney $U$ and Kruskal-Wallis tests for non-parametric data, where appropriate. Categorical data was assessed by Pearson's chi-square test and Fisher's exact test, where appropriate. Univariate analysis was performed to assess the associations between potential predictive factors and whether repeat BCS achieved an adequate margin. Odds ratios (ORs) for failure of repeat BCS were calculated 
for each variable. Variables found to affect the success of repeat BCS in univariate analysis, with a threshold of $\mathrm{p}<0.10$, were included in a multivariate binary logistic regression analysis, with a significance threshold of $\mathrm{p}<0.05$.

Strengthening the reporting of observational studies in epidemiology (STROBE) reporting guidelines were followed when reporting this study.

\section{Results}

One thousand one hundred thirty-four patients underwent initial BCS during the study period. Two hundred twenty (16.5\%) of these underwent reoperation for inadequate margins. Sixty-six underwent mastectomy, leaving 154 patients undergoing repeat BCS. These 154 patients formed the study group. All patients had ultrasonography and mammography, and seven had MRI. Not all patients with a lobular element had preoperative MRI, as the core biopsy had suggested ductal carcinoma, but the final pathology confirmed mixed ductal and lobular carcinoma. The cohort's surgical treatment is shown in Figure 1. One hundred twenty-six patients (82\%) had successful repeat BCS, 104 who had no residual disease and 22 who had residual disease but adequate excision margins. One patient had a successful third BCS, while 27 patients underwent mastectomy as a third procedure.

Thirty-two patients $(21 \%)$, including one with Paget's disease of the breast, had pure DCIS and were excluded from analyses on ER and HER2 status, lymphovascular invasion, and grade. All 122 patients

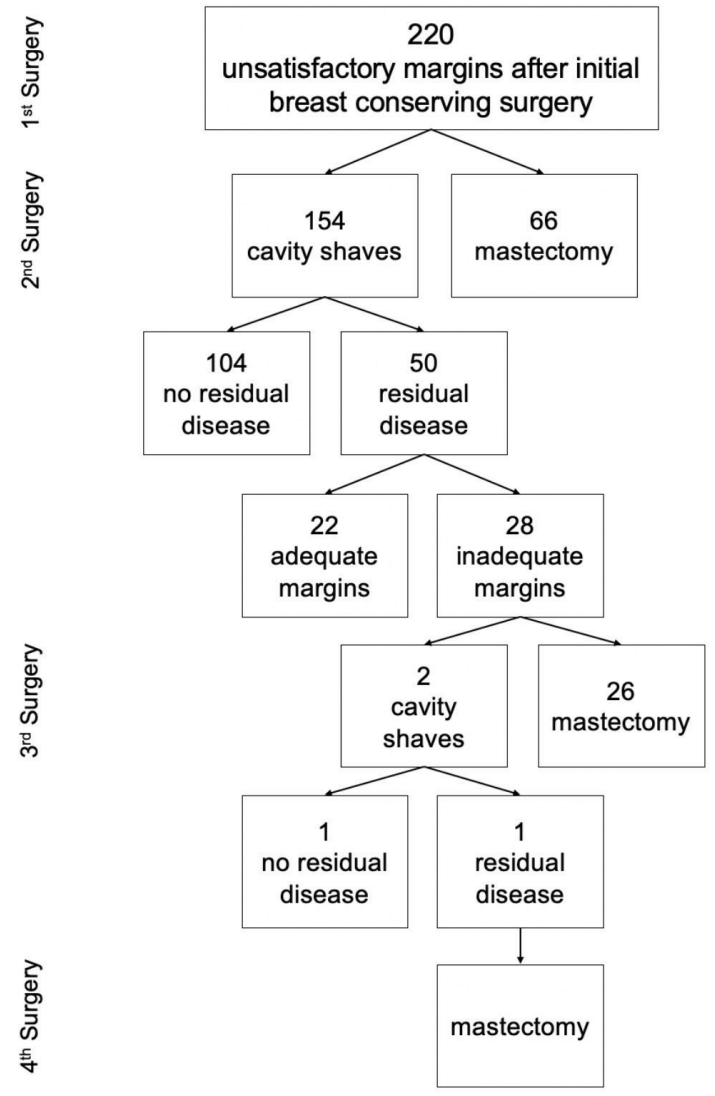

Figure 1. Study group assignment by surgical treatment. In all, $16.5 \%$ of patients had a second operation after initial BCS, $2.1 \%$ had a third operation, and 1 patient $(0.07 \%)$ underwent a fourth

$B C S$ : Breast-conserving surgery with invasive disease had lymph node excision, 16 by axillary clearance and 106 by sentinel node biopsy. The patient with Paget's disease also had sentinel node biopsy.

Patient characteristics and pathological factors for the groups with successful and unsuccessful BCS are shown in Table 1. Patients with successful repeat BCS had smaller tumors and a lower PRR and were less likely to have had targeted cavity shaves taken at their initial BCS. They also tended to be of a lower grade, but this trend did not reach significance. The success rate of repeat BCS decreased as the degree of tumor size underestimation by radiology increased (Figure 2).

Ninety-four percent of patients with two or three of the factors predicting successful repeat BCS did have successful repeat BCS, whereas only $61 \%$ of patients with none of these factors had successful repeat BCS (OR for failure with no factors predicting success was 9.58, 95\% CI: 2.94-31.21, $\mathrm{p}=0.0001$ ).

Univariate and multivariate analyses for failure of repeat BCS are shown in Table 2. Underestimation of tumor size by radiology, with a PRR of over 1.5 , independently predicted failure of repeat BCS in multivariate analysis.

Underestimation of tumor size by radiology was commoner in patients whose specimens contained DCIS. The average PRR was 1.21 in patients with invasive disease only and 1.99 in patients with DCIS ( $\mathrm{p}$ $=0.00398$ ). Eighty-eight percent of patients with invasive disease had a PRR below 1.5 , whereas only $54 \%$ of patients with DCIS did ( $\mathrm{p}=$ 0.00266).

Underestimation of tumor size by radiology was more likely as the pathological tumor size increased (Figure 3). Targeted cavity shaves were more likely to have been taken at the initial BCS where the tumor size was underestimated by radiology $(\mathrm{p}=0.00988$, Figure 4).

Tumor size measurement was similar between mammography and ultrasonography (mean $15.5 \mathrm{~mm}$ vs $16.2 \mathrm{~mm}, \mathrm{p}=0.271$ ). In the seven patients undergoing MRI, the mean MRI tumor measurement was larger than that in the cohort as a whole at $26.3 \mathrm{~mm}$, but these seven patients also had larger tumor measurements on mammography (mean $26 \mathrm{~mm}, \mathrm{p}=0.95)$ and ultrasonography (mean $21 \mathrm{~mm}, \mathrm{p}=0.41)$.

There was no difference in the degree of underestimation of tumor size between mammography and ultrasonography, with a mean PRR of 1.91 for mammography and 1.92 for ultrasonography $(p=0.60)$.

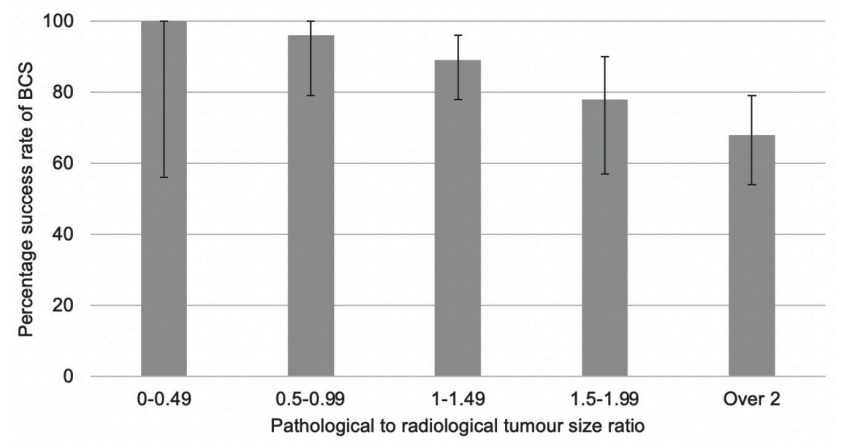

Figure 2. Success rate of repeat BCS by PRR. Repeat BCS was less likely to be successful in patients with a higher degree of tumor size underestimation by radiology

BCS: Breast-conserving surgery, PRR: Pathological tumor size to radiological tumor size ratio 
In the small number of patients undergoing MRI, the mean PRR calculated on the basis of the MRI measurement was lower at 1.48, corresponding to a lesser degree of tumor size underestimation, but this was not significant $(\mathrm{p}=0.234$ vs mammogram and $\mathrm{p}=0.238$ vs ultrasound).

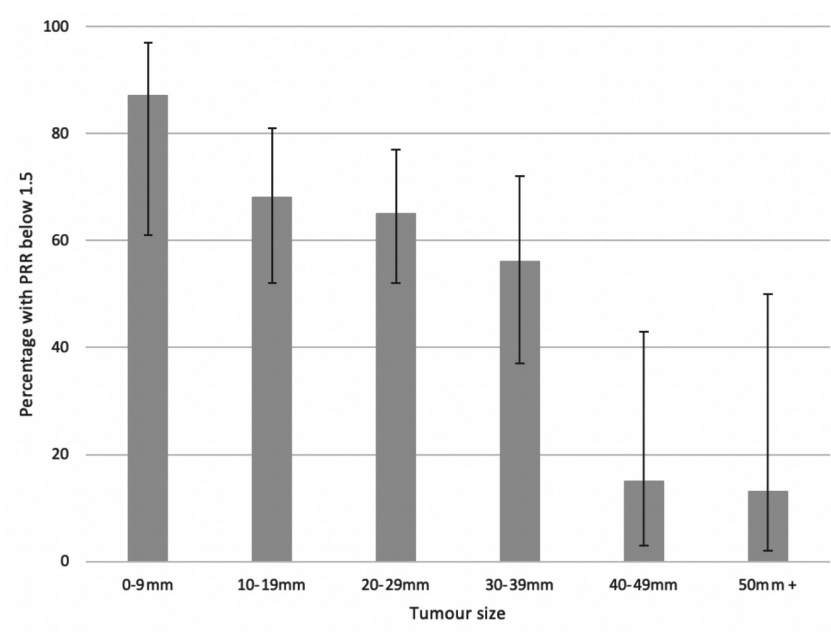

Figure 3. PRR by pathological tumor size. Patients with larger tumors were more likely to have tumor size underestimation by radiology

PRR: Pathological tumor size to radiological tumor size ratio

\section{Discussion and Conclusion}

This study focused on the common problem of re-excision after BCS, in particular the method of that re-excision. We found a high rate of success of repeat BCS but showed that underestimation of the tumor size by imaging independently predicted failure.

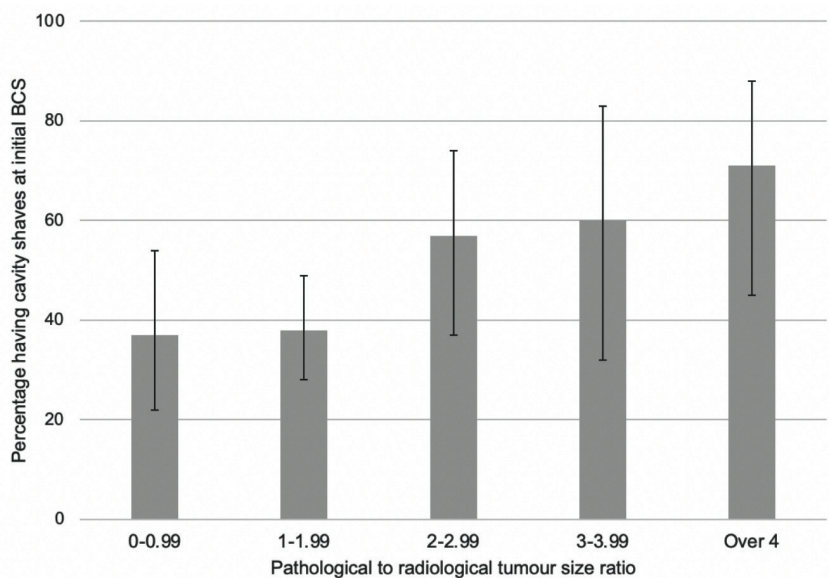

Figure 4. Rate of cavity shaves taken at initial BCS by PRR. Tumor size underestimation by radiology made it more likely that surgeons took unplanned targeted shaves at initial BCS

BCS: Breast-conserving surgery, PRR: Pathological tumor size to radiological tumor size ratio

Table 1. Comparison of patients with successful and unsuccessful repeat BCS

\section{Factor}

Mean age (range)

Any DCIS present

Pure DCIS

Mean radiological tumor size (range)

Mean pathological tumor size (range)

Mean PRR (range)

Multifocal tumor present

IDC

Mean specimen weight (range)

Grade

1

2

3

ER positive

HER2 negative

LVI present

Involved nodes present

Mean number involved margins (range)

Any involved margin

Targeted shaves taken at initial surgery

\section{Successful \\ 127 patients}

$56(32-83)$
$105 / 127(83 \%)$
$27 / 127(21 \%)$
$16 \mathrm{~mm}(3-40)$
$23 \mathrm{~mm}(2-75)$
$1.72(0.3-8)$
$12 / 127(9 \%)$
$91 / 100(91 \%)$
$50 \mathrm{~g} \mathrm{(5-164)}$

$25 / 100(25 \%)$

$47 / 100(47 \%)$

$28 / 100(28 \%)$

$87 / 100(87 \%)$

$85 / 100(85 \%)$

$33 / 100(33 \%)$

$32 / 101(32 \%)$

$0.55(0-2)$

$56 / 127(44 \%)$

$52 / 127(41 \%)$

\section{Unsuccessful \\ 27 patients}

$\begin{array}{cc}59(46-79) & 0.1172 \\ 25 / 27(93 \%) & 0.2532 \\ 5 / 27(19 \%) & 0.7499 \\ 14 \mathrm{~mm} \mathrm{(2-27)} & 0.3030 \\ 29 \mathrm{~mm}(6-50) & 0.0274 \\ 2.56(0.9-7) & 0.0005 \\ 3 / 27(11 \%) & 0.7194 \\ 21 / 22(95 \%) & 0.6881 \\ 51 \mathrm{~g}(11-140) & 0.6312\end{array}$

$2 / 22(9 \%)$

$10 / 22(45 \%)$

$10 / 22$ (45\%)

$19 / 22(86 \%)$

$17 / 22(77 \%)$

0.9361

0.3754

$6 / 22(27 \%)$

0.6020

$6 / 22(27 \%)$

0.6891

$0.74(0-2)$

0.2713

$15 / 27$ (56\%)

0.2780

17/27 (63\%)

DCIS: Ductal carcinoma in situ, PRR: Pathological tumor size to radiological tumor size ratio, IDC: Infiltrating ductal carcinoma, ER: Estrogen receptor, HER2: 
Table 2. Univariate and multivariate analysis of factors predicting failure of repeat BCS

\begin{tabular}{|c|c|c|c|c|c|c|c|}
\hline \multirow[b]{2}{*}{ Factor } & \multirow{2}{*}{$\begin{array}{l}\text { Failure rate of } \\
\text { BCS }\end{array}$} & \multicolumn{3}{|c|}{ Univariate analysis } & \multicolumn{3}{|c|}{ Multivariate analysis } \\
\hline & & $\begin{array}{l}\text { OR for BCS } \\
\text { failure }\end{array}$ & $95 \% \mathrm{Cl}$ & p-value & $\begin{array}{l}\text { OR for BCS } \\
\text { failure }\end{array}$ & $95 \% \mathrm{Cl}$ & p-value \\
\hline \multicolumn{8}{|l|}{ Age } \\
\hline$\geq 50$ & $19 \%$ & 1 & & & & & \\
\hline$<50$ & $14 \%$ & 0.67 & $0.25-1.79$ & 0.4201 & & & \\
\hline \multicolumn{8}{|l|}{ DCIS } \\
\hline Present & $8 \%$ & 1 & & & & & \\
\hline Absent & $19 \%$ & 2.62 & $0.58-11.88$ & 0.2532 & & & \\
\hline \multicolumn{8}{|l|}{ Pure DCIS } \\
\hline Yes & $18 \%$ & 1 & & & & & \\
\hline No & $16 \%$ & 0.84 & $0.29-2.43$ & 0.7518 & & & \\
\hline \multicolumn{8}{|l|}{ Tumor size } \\
\hline$<20 \mathrm{~mm}$ & $9 \%$ & 1 & & & & & \\
\hline$\geq 20 \mathrm{~mm}$ & $22 \%$ & 2.67 & $0.95-7.53$ & 0.0554 & 2.10 & $0.71-6.20$ & 0.178 \\
\hline \multicolumn{8}{|l|}{ PRR } \\
\hline$\leq 1.5$ & $8 \%$ & 1 & & & & & \\
\hline$>1.5$ & $29 \%$ & 4.34 & $1.71-11.03$ & 0.0011 & 3.60 & $1.41-9.20$ & 0.007 \\
\hline \multicolumn{8}{|c|}{ Multifocal tumor } \\
\hline No & $17 \%$ & 1 & & & & & \\
\hline Yes & $20 \%$ & 1.20 & $0.31-4.57$ & 0.7283 & & & \\
\hline \multicolumn{8}{|l|}{ Tumor type } \\
\hline IDC & $19 \%$ & 1 & & & & & \\
\hline ILC or mixed & $10 \%$ & 0.48 & $0.06-4.01$ & 0.6881 & & & \\
\hline \multicolumn{8}{|l|}{ Grade } \\
\hline 1 & $7 \%$ & 1 & & & & & \\
\hline 2 & $18 \%$ & 2.66 & $0.54-13.09$ & 0.3216 & & & \\
\hline 3 & $26 \%$ & 4.46 & $0.89-22.36$ & 0.1020 & & & \\
\hline \multicolumn{8}{|l|}{ LVI } \\
\hline Absent & $19 \%$ & 1 & & & & & \\
\hline Present & $15 \%$ & 0.76 & $0.27-2.13$ & 0.6033 & & & \\
\hline \multicolumn{8}{|c|}{ Axillary nodes } \\
\hline Not involved & $19 \%$ & 1 & & & & & \\
\hline Involved & $16 \%$ & 0.81 & $0.29-2.26$ & 0.6892 & & & \\
\hline \multicolumn{8}{|c|}{ Any margin involved } \\
\hline No & $14 \%$ & 1 & & & & & \\
\hline Yes & $21 \%$ & 1.58 & $0.69-3.66$ & 0.2774 & & & \\
\hline \multicolumn{8}{|c|}{ Margins involved } \\
\hline 0 & $14 \%$ & 1 & & & & & \\
\hline 1 & $19 \%$ & 1.41 & $0.56-3.54$ & 0.4666 & & & \\
\hline 2 & $26 \%$ & 2.11 & $0.64-6.95$ & 0.3024 & & & \\
\hline \multicolumn{8}{|c|}{ Any targeted shaves taken at initial BCS } \\
\hline Yes & $12 \%$ & 1 & & & & & \\
\hline No & $25 \%$ & 2.45 & $1.04-5.78$ & 0.0368 & 1.91 & $0.77-4.71$ & 0.162 \\
\hline
\end{tabular}


Table 2. Continued

\begin{tabular}{|c|c|c|c|c|c|c|c|}
\hline \multirow{2}{*}{ Factor } & \multirow{2}{*}{$\begin{array}{l}\text { Failure rate of } \\
\text { BCS }\end{array}$} & \multicolumn{3}{|c|}{ Univariate analysis } & \multicolumn{3}{|c|}{ Multivariate analysis } \\
\hline & & $\begin{array}{l}\text { OR for BCS } \\
\text { failure }\end{array}$ & $95 \% \mathrm{Cl}$ & p-value & $\begin{array}{l}\text { OR for BCS } \\
\text { failure }\end{array}$ & $95 \% \mathrm{Cl}$ & p-value \\
\hline \multicolumn{8}{|l|}{ ER status } \\
\hline Positive & $18 \%$ & 1 & & & & & \\
\hline Negative & $19 \%$ & 1.06 & $0.27-4.08$ & 0.9361 & & & \\
\hline \multicolumn{8}{|c|}{ HER2 status } \\
\hline Negative & $17 \%$ & 1 & & & & & \\
\hline Positive & $25 \%$ & 1.67 & $0.53-5.20$ & 0.5242 & & & \\
\hline
\end{tabular}

Re-excision is currently a widely debated topic in breast surgery. Substantial efforts have been made in recent times to reduce rates of re-excision. Novel surgical techniques, including intraoperative ultrasound, intraoperative cytology, in-theater specimen radiology, and circumferential cavity shaving, have been introduced to reduce margin involvement $(9,10)$. Much work has also been carried out investigating the size of the resection margin that gives the optimum balance between unnecessary re-excision and future local recurrence. While debate remains over what constitutes an adequate margin, with United Kingdom (UK) guidelines recommending a $1 \mathrm{~mm}$ margin for invasive disease, while United States (US) guidelines mandate only no tumor on ink, it is clear that avoiding involved margins is essential in reducing the tumor burden sufficiently so that the combination of surgery and adjuvant therapy can lead to extremely low local recurrence rates (11). The ideal scenario would clearly be to achieve this at initial BCS; however, if this is not achieved, re-excision still reduces local recurrence, although possibly not to the same level as if adequate margins were achieved at the initial BCS, particularly if the re-excision contains residual disease (12-14). Inaccurate targeting of re-excision may at least partially explain this. Particularly with mobilization of glandular flaps to fill the lumpectomy defect at initial BCS, the exact site of margin involvement may not be correctly identified at repeat BCS, potentially leaving residual disease in the breast despite a histologically clear re-excision specimen. The sharing of adverse prognostic indicators between the need for re-excision and local recurrence may also contribute. While local recurrence rates may be higher, overall survival in patients undergoing re-excision is no different to those having successful initial BCS, whether the reexcision is achieved by repeat BCS or mastectomy (15). Although reexcision rates are improving, with a meta-analysis finding a re-excision rate of $14 \%$ in recent studies, substantially lower than historic rates, the burden of re-excision remains high (2). Given how frequently the decision on the method of re-excision needs to be made, very few studies have looked at the rate of success of re-excision BCS or investigated the factors that influence it. Our study showed a success rate of re-excision BCS of $82 \%$. It must be borne in mind that this was a group of patients considered appropriate candidates for repeat BCS, and $30 \%$ of patients with inadequate margins during the study period chose mastectomy as their method of first re-excision and so were not included in this study group. Fisher et al. also showed a success rate for repeat BCS of $82 \%$, Morrow et al. (16) 93\%, and Coopey et al. (17) factors influencing the success of repeat BCS (15-17). Houvenaeghel et al. (18) showed a success rate of repeat BCS of $87 \%$, with patients under 50 and those with larger or multifocal tumors less likely to have successful repeat BCS. In a cohort of patients with invasive lobular carcinoma, Piper et al. found a success rate of repeat BCS of 74\%, with higher success rates in those who were older and had fewer involved nodes. Patients whose repeat BCS was unsuccessful also had larger tumors in their study, but this did not reach significance (19).

This study did not investigate the type of re-excision to offer if repeat BCS did not achieve adequate margins. In our study, all but two patients in this situation underwent mastectomy. Our policy is to avoid more than three operations on the breast, if possible, based on concerns regarding excess tumor burden, delay to adjuvant therapy, and previous national guidance. Of the two patients who underwent a third BCS in our cohort, one achieved adequate margins, while the other underwent mastectomy as a fourth operation. Other series have addressed this situation, with Cellini et al. (20) showing a 61\% success rate and Coopey et al. (17) a 67\% success rate at third BCS and 25\% at fourth BCS, with a $2 \%$ local recurrence rate in those patients at 64-month median follow-up.

Underestimation of tumor size by radiology is a well-recognized problem in the literature. It has previously been shown that radiological tumor size underestimation influences the success of initial BCS, with a greater degree of underestimation leading to a greater need for reexcision (21). Tumor size underestimation has also been shown to increase the probability of residual disease in the re-excision specimen (22). We showed that a pathological tumor size exceeding the radiological measurement by more than $50 \%$ independently predicted a higher failure rate of repeat BCS, to our knowledge the first study to demonstrate this in the literature. The rate of underestimation was generally high in this study as it included only patients who had failed initial BCS, a group known to have a higher rate of underestimation (21). The imaging modality may play a role in tumor size underestimation, having previously been shown to be commoner with ultrasonography than with mammography (23). Ultrasonography is operator dependent, and underestimation may be due to factors such as failure to measure the halo around the tumor or the tumor size exceeding the size of the transducer. In tumors with a significant component of DCIS, the tumor extent may be underestimated on ultrasound as the typical microcalcifications are less readily visible or measurable. Non- 
calcified DCIS may lead to tumor underestimation on mammography (24-26). We found no difference in the degree of size underestimation between mammography and ultrasonography in this study, although we did find that underestimation of tumor size was commoner in patients with DCIS and also with larger tumors, findings echoed in other studies $(25,27)$. Radiological underestimation of tumor size has been shown to occur less often with MRI, although MRI can also lead to overestimation, possibly due to enhancement of background parenchyma $(24,28)$. We found that MRI underestimated tumor size to a lesser extent than mammography or ultrasound, although too few patients in this cohort underwent MRI to allow a useful comparison.

The pathological tumor type also influences the degree of radiological size underestimation. Lobular primaries are at higher risk of radiological underestimation, due to their diffuse growth pattern, with less distortion of the breast architecture and a lack of difference in density or echogenicity between the tumor and normal breast tissue. Lobular tumors are also more likely to exhibit irregular contours and more diffuse margins and have a higher likelihood of satellite foci (29). We did not find a higher rate of underestimation in lobular tumors, although they made up only 10 patients of our cohort.

We found that patients with a greater degree of radiological underestimation were more likely to have had targeted cavity shaves taken at the time of their initial BCS. We believe this is because the surgeon's initial excision is guided by the preoperative radiological tumor size, with a larger tumor than expected only being detected intraoperatively, by direct palpation, visualization, or intraoperative specimen radiology and leading to additional tissue being taken. To our knowledge, this is the first time this correlation has been reported in the literature.

A limitation of the study is that patients were from a single center, which limited the number of patients, and the treatment decisions made may not be replicated in other centers. We followed UK guidelines on an adequate margin distance of $1 \mathrm{~mm}$, different to US and other European guidelines, which may make this study less applicable in countries following those guidelines. A further limitation is that we had no data on cosmetic outcomes for patients who had reexcision, relying on margin adequacy as the only marker for success of the repeat BCS.

Further work could explore the extent to which patients value particular factors, such as the risk of additional surgery, cosmesis, delay in adjuvant treatment, or potential avoidance of radiotherapy when making the decision on whether to have repeat BCS or to choose mastectomy.

In conclusion, re-excision by cavity shave has a high success rate and should be offered to all patients who are thought suitable. Patients whose tumors are more than $50 \%$ larger than was predicted on imaging should be counseled about the higher risk of failure with consideration given to larger excisions or oncoplastic techniques.

Ethics Committee Approval: The authors state that the study protocol has been approved by the Northern Health and Social Care Trust research committee (decision number: NT20-274636-02 date: June 10th, 2020).

Informed Consent: Patient-level consent was waived by the committee as no treatment decisions were altered by the study.

Peer-review: Externally peer-reviewed.
Authorship Contributions: Surgical and Medical Practices: D.S., J.A., B.M.; Concept: D.S., B.M.; Design: D.S., B.M.; Data Collection and/or Processing: D.S., J.A., B.M.; Literature Search: D.S., J.A., B.M.; Writing: D.S., J.A., B.M.

Conflict of Interest: No conflict of interest was declared by the authors.

Financial Disclosure: The authors declare that this study received no financial support.

\section{References}

1. Veronesi U, Cascinelli N, Mariani L, Greco M, Saccozzi R, Luini A, et al. Twenty-year follow-up of a randomized study comparing breastconserving surgery with radical mastectomy for early breast cancer. $\mathrm{N}$ Engl J Med 2002; 347: 1227-1232. (PMID: 1212393819) [Crossref]

2. Havel L, Naik H, Ramirez L, Morrow M, Landercasper J. Impact of the SSO-ASTRO margin guideline on rates of re-excision after lumpectomy for breast cancer: a meta-analysis. Ann Surg Oncol 2019; 26: 1238-1244. (PMID: 30790112) [Crossref]

3. Cody HS, Van Zee KJ. Reexcision - the other breast cancer epidemic. N Engl J Med 2015; 373: 568-569. (PMID: 26244311) [Crossref]

4. Kim MK, Kim T, Moon HG, Jin US, Kim K, Kim J, et al. Effect of cosmetic outcome on quality of life after breast cancer surgery. Eur J Surg Oncol 2015; 41: 426-432. (PMID: 25578249) [Crossref]

5. Ng ET, Ang RZ, Tran BX, Ho CS, Zhang Z, Tan W, et al. Comparing quality of life in breast cancer patients who underwent mastectomy versus breast-conserving surgery: a meta-analysis. Int J Environ Res Public Health 2019; 16: 4970. (PMID: 31817811) [Crossref]

6. Heil J, Breitkreuz K, Golatta M, Czink E, Dahlkamp J, Rom J, et al. Do reexcisions impair aesthetic outcome in breast conservation surgery? Exploratory analysis of a prospective cohort study. Ann Surg Oncol 2012; 19: 541-547. (PMID: 21761099) [Crossref]

7. Olsen MA, Nickel KB, Margenthaler JA, Wallace AE, Mines D, Miller JP, et al. Increased risk of surgical site infection among breast-conserving surgery re-excisions. Ann Surg Oncol 2015; 22: 2003-2009. (PMID: 25358666) [Crossref]

8. Grant Y, Al-Khudairi R, St John E, Barschkett M, Cunningham D, Al-Mufti R, et al. Patient-level costs in margin re-excision for breastconserving surgery. Br J Surg 2019; 106: 384-394. (PMID: 30566233) [Crossref]

9. Angarita FA, Nadler A, Zerhouni S, Escallon J. Perioperative measures to optimize margin clearance in breast conserving surgery. Surg Oncol 2014; 23: 81-91. (PMID: 24721660) [Crossref]

10. Wang $\mathrm{K}$, Ren Y, He J. Cavity shaving plus lumpectomy versus lumpectomy alone for patients with breast cancer undergoing breastconserving surgery: a systematic review and meta-analysis. PloS One 2017; 12: e0168705. (PMID: 28046058) [Crossref]

11. Houssami N, Macaskill P, Marinovich ML, Morrow M. The association of surgical margins and local recurrence in women with early-stage invasive breast cancer treated with breast-conserving therapy: a meta-analysis. Ann Surg Oncol 2014; 21: 717-730. (PMID: 24473640) [Crossref]

12. Vos EL, Siesling S, Baaijens MHA, Verhoef C, Jager A, Voogd AC, et al. Omitting re-excision for focally positive margins after breast-conserving surgery does not impair disease-free and overall survival. Breast Cancer Res Treat 2017; 164: 157-167. (PMID: 28389735) [Crossref]

13. Tang SSK, Rapisarda F, Nerurkar A, Osin P, MacNeill F, Smith I, et al. Complete excision with narrow margins provides equivalent local control to wider excision in breast conservation for invasive cancer. BJS Open 2019; 3: 161-168. (PMID: 30957062) [Crossref]

14. Hennigs A, Fuchs V, Sinn H-P, Riedel F, Rauch G, Smetanay K, et al. Do patients after reexcision due to involved or close margins have the same risk of local recurrence as those after one-step breast-conserving surgery? Ann Surg Oncol 2016; 23: 1831-1837. (PMID: 26732272) [Crossref] 
15. Fisher S, Yasui Y, Dabbs K, Winget M. Re-excision and survival following breast conserving surgery in early stage breast cancer patients: a population-based study. BMC Health Serv Res 2018; 18: 94. (PMID: 29422097) [Crossref]

16. Morrow M, Abrahamse P, Hofer TP, Ward KC, Hamilton AS, Kurian AW, et al. Trends in Reoperation After Initial Lumpectomy for Breast Cancer: Addressing Overtreatment in Surgical Management. JAMA Oncol 2017; 3: 1352. (PMID: 28586788) [Crossref]

17. Coopey S, Smith BL, Hanson S, Buckley J, Hughes KS, Gadd M, et al. The safety of multiple re-excisions after lumpectomy for breast cancer. Ann Surg Oncol 2011; 18: 3797-3801. (PMID: 21630123) [Crossref]

18. Houvenaeghel G, Lambaudie E, Bannier M, Rua S, Barrou J, Heinemann $\mathrm{M}$, et al. Positive or close margins: reoperation rate and second conservative resection or total mastectomy? Cancer Manag Res 2019; 11: 2507-2516. (PMID: 30992681) [Crossref]

19. Piper ML, Wong J, Fahrner-Scott K, Ewing C, Alvarado M, Esserman $\mathrm{LJ}$, et al. Success rates of re-excision after positive margins for invasive lobular carcinoma of the breast. NPJ Breast Cancer 2019; 5: 29. (PMID: 31508489) [Crossref]

20. Cellini C, Huston TL, Martins D, Christos P, Carson J, Kemper S, et al. Multiple re-excisions versus mastectomy in patients with persistent residual disease following breast conservation surgery. Am J Surg 2005; 189: 662-666. (PMID: 15910716) [Crossref]

21. Dixon JM, Newlands C, Dodds C, Thomas J, Williams LJ, Kunkler IH, et al. Association between underestimation of tumour size by imaging and incomplete excision in breast-conserving surgery for breast cancer. $\mathrm{Br} \mathrm{J}$ Surg 2016; 103: 830-838. (PMID: 27171027) [Crossref]

22. Mimouni M, Lecuru F, Rouzier R, Lotersztajn N, Heitz D, Cohen J, et al. Reexcision for positive margins in breast cancer: a predictive score of residual disease. Surg Oncol 2015;24:129-135. (PMID: 26298198) [Crossref]
23. Khalayleh H, Khalayleh M, Diment J, Allweis TM. Breast density does not affect breast cancer tumor size assessment: a comparison of radiologic versus pathologic measurement by different imaging modalities across breast densities. Eur J Surg Oncol 2020; 46: 1435-1440. (PMID: 32115332) [Crossref]

24. Daniel OK, Lim SM, Kim JH, Park HS, Park S, Kim SI. Preoperative prediction of the size of pure ductal carcinoma in situ using three imaging modalities as compared to histopathological size: does magnetic resonance imaging add value? Breast Cancer Res Treat 2017; 164: 437-444. (PMID: 28439735)

25. Gruber IV, Rueckert M, Kagan KO, Staebler A, Siegmann KC, Hartkopf A, et al. Measurement of tumour size with mammography, sonography and magnetic resonance imaging as compared to histological tumour size in primary breast cancer. BMC Cancer 2013; 13: 328. (PMID: 23826951) [Crossref]

26. Kuhl CK, Lehman C, Bedrosian I. Imaging in locoregional management of breast cancer. J Clin Oncol 2020; 38: 2351-2361. (PMID: 32442068) [Crossref]

27. Cuesta Cuesta AB, Martín Ríos MD, Noguero Meseguer MR, García Velasco JA, Matías Martínez M de, Bartolomé Sotillos S, et al. Accuracy of tumor size measurements performed by magnetic resonance, ultrasound and mammography, and their correlation with pathological size in primary breast cancer. Cir Esp Engl Ed 2019; 97: 391-396. (PMID: 31186117) [Crossref]

28. Choi WJ, Cha JH, Kim HH, Shin HJ, Chae EY. The accuracy of breast MR imaging for measuring the size of a breast cancer: analysis of the histopathologic factors. Clin Breast Cancer 2016; 16: e145-e152. (PMID: 27566960) [Crossref]

29. Malherbe K, Bresser P. Association between ultrasound morphologic features and histopathological findings of lobular carcinoma. J Med Radiat Sci 2019; 66: 177-183. (PMID: 31472006) [Crossref] 